Pure and Applied Mathematics Quarterly

Volume 1, Number 4

(Special Issue: In Memory of

Armand Borel, Part 3 of 3 )

$867-888,2005$

\title{
A Conjecture about the Analytical Behaviour of Eisenstein Series
}

\author{
Bill Casselman
}

This paper will formulate and offer evidence for a conjecture on the analytical behaviour of residual Eisenstein series in the positive Weyl chamber. It should play a major role in an also conjectural Paley-Wiener theorem about the Schwartz space of arithmetic quotients. If things work out as I expect, this development should be logically independent of Chapter 7 of [Langlands:1976], and in the end one should obtain a new proof of Langlands' completeness theorem.

Suppose $G$ to be the group of real points on a reductive group defined over $\mathbb{Q}$ and further set

$\begin{array}{ll}\Gamma & \text { an arithmetic subgroup of } G \\ K & \text { a maximal compact subgroup of } G \\ Z_{G} & \text { the centre of } G\end{array}$

For a rational parabolic subgroup $P$, set

$$
\begin{array}{ll}
N=N_{P} & \text { its unipotent radical } \\
M=M_{P} & \text { its reductive quotient } P / N_{P} \\
\Gamma_{M} & \text { the image of } \Gamma \cap P \text { in } M \\
A_{P} & \text { the maximal } \mathbb{Q} \text {-split torus in } M \\
\delta_{P} & \text { its modulus character } \operatorname{det}_{A d_{n}}
\end{array}
$$

If $\left|A_{P}\right|$ is the connected component of $A_{P}$, then $M$ is as an analytic group equal to the direct product of $\left|A_{P}\right|$ with a subgroup of $M$ which contains the image $\Gamma_{M}$ of $\Gamma \cap P$ in $M$. There are unique liftings of both $A_{P}$ and $M_{P}$ to $P$ stable under the Cartan involution of $G$ determined by the choice of $K$, which I'll also call $A_{P}$ and $M_{P}$. The group $\left|A_{P}\right|$ may then be identified with $A_{P} / K \cap A_{P}$. The characters $\delta_{Q}$ for $Q$ a proper maximal rational parabolic subgroup containing $P$ make up a basis of the characters of $\left|A_{P}\right| /\left|A_{G}\right|$, considered as a complex vector space. Let the dual basis in the group $\left|A_{P}\right| /\left|A_{G}\right|$ be $\left\{a_{Q}\right\}$, identified with elements of $P$.

Received August 17, 2005. 
Let $X(P)$ be the set of unramified characters of $P$, those trivial on $K \cap P$, $\Gamma \cap P$, and (necessarily) $N$. Because of well known density results, it is equivalent to require that such a character factor through projection onto $\left|A_{P}\right|$. The space $X(P)$ may be identified with a Euclidean space - if $\left(a_{i}\right)$ is an isomorphism of $\left|A_{P}\right|$ with a product of copies of the positive real numbers, then the correspondence takes $\left(z_{i}\right)$ to $\prod a_{i}^{z_{i}}$. The space of unitary characters becomes thus a real Euclidean space; choose a Euclidean norm on it. For any character $\chi,\|\operatorname{IM}(\chi)\|$ is the norm of the unitary character $\chi /|\chi|$.

Because $G=P K$, any unramified character $\chi$ of $P$ extends to a unique function on all of $G$ fixed by right $K$-multiplication, one which I'll also express as $\chi$. This applies in particular to the function $\delta_{P}$. The positive cone in the vector space $X(P)$ is the subset $X^{++}(P)$ where all $\left|\chi\left(a_{Q}\right)\right|>1$. If

$$
d(\chi)=\prod\left(\left|\chi\left(a_{Q}\right)\right|-1\right)
$$

then the boundary of this cone is where $d(\chi)=0$.

The space $\mathcal{A}(N(\Gamma \cap P) \backslash G)$ of automorphic forms on $N(\Gamma \cap P) \backslash G$ is that of all smooth $\mathbb{C}$-valued functions $\varphi$ on $G$ which are (a) right $K$-finite; (b) $Z$ (g)-finite; (c) left $Z_{M}$-finite; and (d) which satisfy in addition a condition of moderate growth I won't specify precisely. This space may be identified with the representation of $G$ induced from that of $P$ on the space $\mathcal{A}\left(\Gamma_{M} \backslash M\right)$. If $\varphi$ is a function in this space such that $\varphi(z g)=\chi(z) \delta_{P}^{1 / 2}(z) \varphi(g)$ for some unitary character $\chi$ of the centre $Z_{M}$ of $M_{P}$, then $|\varphi|^{2}$ lies in the space $\Omega\left(N_{P} Z_{M}(\Gamma \cap P) \backslash G\right)$ of onedensities on $N Z_{M}(\Gamma \cap P) \backslash G$. Define $\mathcal{A}^{2}\left(N_{P}(\Gamma \cap P) \backslash G\right)$ to be the space of all such functions $\varphi$ such that $|\varphi|^{2}$ is integrable. In particular $|\varphi(a g)|=\delta^{1 / 2}(a)|\varphi(g)|$ for any function in this space and all $a$ in $\left|A_{P}\right|$. This space is also induced, here from the automorphic forms on $\Gamma_{M} \backslash M$ that are square-integrable modulo $Z_{M}$.

Recall from [Casselman:1989] that the Schwartz space $\mathfrak{S}(\Gamma \backslash G)$ is that of all $K$-finite functions all of whose $U(\mathfrak{g})$-derivatives decrease more rapidly than any character on Siegel sets. (This is what [Franke:1998] calls $S_{-\infty}$.) For $\varphi$ in the space $\mathcal{A}^{2}\left(N_{P}(\Gamma \cap P) \backslash G\right)$ the Eisenstein series

$$
E_{\varphi \chi}(g)=\sum_{\Gamma \cap P \Gamma} \delta_{P}^{1 / 2}(\gamma g) \chi(\gamma g) \varphi(\gamma g)
$$

converges in some open cone of $X(P)$ and extends to a meromorphic function on all of $X(P)$. For $f$ in $\mathfrak{S}(\Gamma \backslash G)$ the Fourier-Eisenstein integral

$$
\widehat{f}_{\varphi}(\chi)=\int_{\Gamma \backslash G} f(g) E_{\varphi \chi^{-1}}(g) d g
$$

defines a meromorphic function of $\chi$. A Paley-Wiener theorem for $\Gamma \backslash G$ would characterize a function in the Schwartz space by such integrals. One fundamental property in a characterization is laid out in the following: 
Conjecture. Suppose $\Omega$ to be any bounded region in the Euclidean space of real unramified characters of $P$. There exists a product $P(s)$ of affine functions on $X(P)$ and some integer $r>0$ such that

$$
P_{\varphi}(s) \widehat{f}\left(\chi_{s}^{-1}\right)=O\left(\frac{1}{(1+\|\operatorname{IM}(\chi)\|)^{N}\|d(\chi)\|^{r}}\right) .
$$

for all $N>0$ and $\operatorname{RE}(s)$ in $\Omega \cap X^{++}(P)$.

The significance of this property will be better understood if one looks at $\S 13$ of [Casselman: 2004]. Roughly speaking, it allows contour motion in the proof of a Paley-Wiener theorem. The Conjecture is somewhat imprecise, since it does not specify conditions on an Eisenstein series independent of its pairing with functions in the Schwartz space. I have such conditions in mind, and I shall in fact exhibit some later on, but I am not so sure of their general validity as I am of the assertion above.

The Conjecture has little direct bearing on the location of the singularities of Eisenstein series. In particular, it says nothing about the square-integrable residues of Eisenstein series, but only about the Eisenstein series constructed from such residues. Indeed, I expect the Conjecture to be established without any explicit information about residues other than square-integrability.

According to the main result of [Franke: 1999], there exists a real polynomial $P(s)$ with $P(s) E_{\varphi \chi}$ holomorphic in $X^{++}(P)$, and this could be used to sharpen the Conjecture. However, I can't imagine proving the Conjecture without simultaneously providing a new proof of Franke's theorem. Furthermore, [Franke: 1999] depends on [Franke: 1998], and since one of my aims is to provide eventually a new proof of many of Franke's results in this earlier paper, I hope to establish the Conjecture independently of them. The Conjecture is also closely related to results in a series of papers by Werner Müller on the trace class conjecture, but I can't see any direct relationship. Again, Müller's work depends to some extent on Franke's, so the same remarks apply to it.

If $\varphi$ lies in the space of cusp forms on $N(\Gamma \cap P) \backslash G$, the Conjecture is implicit in known theorems, as I shall explain to some extent in the next section. For some $\varphi$ that are not cusp forms it is probably implicit in the calculations that have gone into verifying various conjectures of Arthur on the discrete spectrum of $\Gamma \backslash G$. But in the generality I formulate the Conjecture it seems to be new. The importance of the matter can be judged from the fact that much of the difficulty of Langlands' proof of his spectral completeness theorem is caused precisely by his not knowing much about the analytical behaviour of Eisenstein series constructed from residues. Because of this, the contour movements he dealt with were technically intricate. If the Conjecture is true, I expect that his argument 
can be simplified. Well ... perhaps not simplified, but at least redone so as to yield a stronger result, along the lines of the Paley-Wiener theorem demonstrated for $S L_{2}$ in my Shalika volume paper. In addition, the argument I have in mind promises to avoid the peculiar redundancy and occasional cancellation that afflicts Langlands' contour movement.

I have tried to make this paper as self-contained as possible. This will undoubtedly make it verbose for some, but of course they can skip familiar material.

$$
\text { 1. } \mathrm{SL}_{2}(\mathbb{R})
$$

I start with the simplest case. This should make clear the nature of the Conjecture as well as some of the problems involved in proving it, and should also suggest a line of attack on it.

Let $G$ be $\mathrm{SL}_{2}, P$ the subgroup of upper triangular matrices, $\mathcal{H}$ the upper half plane. Take $\Gamma$ to be a discrete subgroup of $G$ with one cusp, located at $\infty$. Of course $\mathrm{SL}_{2}(\mathbb{Z})$ will fit the description, but it is too special to serve as a good example. Conjugating if necessary we may assume $\Gamma \cap N$ to be the group of horizontal integral translations. For $Y \gg 1$ the region $|x| \leq 1 / 2, y \geq Y$ embeds into $\Gamma \backslash \mathcal{H}$ with compact complement. Equivalently, if $\mathcal{H}_{Y}$ is the region where $y>Y$, the projection from $\Gamma \cap N \backslash \mathcal{H}_{Y}$ to $\Gamma \backslash \mathcal{H}$ is an embedding. The Schwartz space $\mathcal{S}(\Gamma \backslash \mathcal{H})$ is that of all smooth functions $f$ such that all $\Delta^{k} f$ are more rapidly decreasing than any $1 / y^{n}$ as $y$ goes to infinity.

If $\alpha$ is the single positive root, the coroot is

$$
\alpha^{\vee}: x \longmapsto\left[\begin{array}{ll}
x & \\
1 / x
\end{array}\right]
$$

For $s$ in $\mathbb{C}$, let $\chi_{s}$ be the character taking $\alpha^{\vee}(x)$ to $|x|^{s}$. The function $y^{1 / 2+s / 2}$ is an eigenfunction for the Laplacian $\Delta$ with eigenvalue $\lambda_{s}=\left(s^{2}-1\right) / 4$. The Eisenstein series we are interested in is the corresponding $\Gamma$-invariant function on $\mathcal{H}:$

$$
E_{s}(z)=\sum_{\Gamma \cap P \backslash \Gamma} y^{1 / 2+s / 2}(\gamma z) \quad(y(z)=\operatorname{IM}(z)),
$$

which converges for $\operatorname{RE}(s)>1$. Its constant term specifies its asymptotic behaviour as $t$ goes to $\infty$, and is explicitly

$$
y^{1 / 2+s / 2}+c(s) y^{1 / 2-s / 2}
$$

for some meromorphic function $c(s)$. When $\Gamma=\mathrm{SL}_{2}(\mathbb{Z})$

$$
c(s)=\Xi(s)=\frac{\xi(s)}{\xi(s+1)},
$$


where $\xi$ is Riemann's function

$$
\xi(s)=\pi^{-s / 2} \Gamma(s / 2) \zeta(s) .
$$

Most of what happens can be indicated in a single diagram, which shows the case $\Gamma=\mathrm{SL}_{2}(\mathbb{Z})$ :

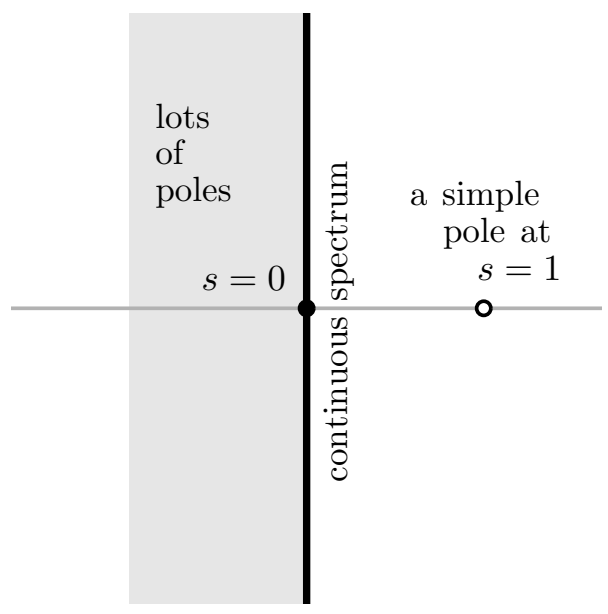

Figure 1. Behaviour of Eisenstein series for $\mathrm{SL}_{2}(\mathbb{Z})$

Here, there is a solitary and simple pole at $s=1$ in the region $\operatorname{RE}(s) \geq 0$, where the residue of the Eisenstein series is the constant function $1 / \xi(2)$. There will be a plethora of more interesting poles at all $s$ with $\xi(s+1)=0$, which are located in the region $-1<\operatorname{RE}(s)<0$. This is a region best left unexplored, at least for now! The continuous spectrum is supported on the imaginary axis $\operatorname{RE}(s)=0$.

Here are two ways to verify the Conjecture in this case, each with its own virtues.

(1) I follow [Langlands:1966]. Truncate the entire constant term at $Y$, getting an orthogonal decomposition

$$
E_{s}=\Lambda^{Y} E_{s}+C^{Y} E_{s} .
$$

The first term is square-integrable for all $s$. Its $\mathrm{L}^{2}$-norm is given by the MaassSelberg formula

$$
\left\|\Lambda^{Y} E_{s}\right\|^{2}=\frac{Y^{\sigma}-|c(s)|^{2} Y^{-\sigma}}{\sigma}-\frac{c(s) Y^{-i t}-\overline{c(s)} Y^{i t}}{i t} \quad(s=\sigma+i t)
$$

for neither $\sigma$ nor $t$ equal to 0 .

Lemma 1.1. In any region where $0<\sigma \leq \sigma_{0}, t \geq t_{0}$ the $c$-function is bounded. In a region where $0<\sigma \leq \sigma_{0}, t \leq t_{0}$ the product $c(s) t$ is bounded.

One consequence is that $c(s)$ has poles in the region $\sigma>0$ only on $(0,1]$, and that those poles are simple. Another is that if those poles are the $s_{i}$ and we set 
$P(s)=\prod\left(s-s_{i}\right)$, for some $N$

$$
\left\|\Lambda^{Y} P(s) E_{s}\right\|=O\left(\frac{|t|^{N}}{\sqrt{\sigma}}\right)
$$

on regions where $\sigma>0$ is bounded.

Proof. The expression in the Maass-Selberg formula must be non-negative. Hence:

$$
\begin{aligned}
\left(Y^{\sigma}-|c(s)|^{2} Y^{-\sigma}\right) & \geq \frac{\sigma}{i t}\left(c(s) Y^{-i t}-\overline{c(s)} Y^{i t}\right) \\
Y^{2 \sigma} & \geq|c(s)|^{2}+\frac{\sigma}{i t}\left(c(s) Y^{\sigma-i t}-\overline{c(s)} Y^{\sigma+i t}\right) \\
Z \bar{Z} & \geq c \bar{c}+\frac{\sigma}{t}(c Z / i+\bar{c} \bar{Z} / \bar{i}) \quad\left(Z=Y^{\sigma-i t}\right) \\
Z \bar{Z}\left(1+\frac{\sigma^{2}}{t^{2}}\right) & \geq c \bar{c}+\frac{\sigma}{t}(c Z / i+\bar{c} \bar{Z} / \bar{i})+Z \bar{Z} \frac{\sigma^{2}}{t^{2}} \\
|Z|\left|1+\frac{\sigma}{i t}\right| & \geq\left|c+\frac{\sigma}{i t} Z\right| \\
\left|1+\frac{\sigma}{i t}\right| & \geq\left|\frac{c}{Z}+\frac{\sigma}{i t}\right| \\
& =\left|\left(\frac{c}{Z}-1\right)+1+\frac{\sigma}{i t}\right|=\left|\left(1-\frac{c}{Z}\right)-\left(1+\frac{\sigma}{i t}\right)\right| \\
\left|1-\frac{c}{Z}\right| & \leq 2\left|1+\frac{\sigma}{i t}\right| .
\end{aligned}
$$

In a region $0<\sigma \leq \sigma_{0}, t \geq t_{0}$ the quotient $\sigma / t$ is bounded, hence so is $c / Z$, But $|Z|=Y^{\sigma}$ will also be bounded, hence $c$ is bounded.

The last equation can be rewritten

$$
\left|t-\frac{t c}{Z}\right| \leq 2\left|1+\frac{\sigma}{i}\right|
$$

and from this it follows that in a region $0<\sigma \leq \sigma_{0}, 0<t \leq t_{0}$ the product $t c$ is bounded.

Now if we take $f$ in the Schwartz space we have

$$
\begin{aligned}
\left\langle\Delta^{k} f, E_{s}\right\rangle & =\lambda_{s}^{k}\left\langle f, E_{s}\right\rangle \\
\left\langle f, E_{s}\right\rangle & =\left\langle f, \Lambda^{Y} E_{s}\right\rangle+\left\langle f, C^{Y} E_{s}\right\rangle \\
& \leq\|f\|\left\|\Lambda^{Y} E_{s}\right\|+\int_{Y}^{\infty} f_{0}(y)\left(y^{1 / 2+s / 2}+c(s) y^{1 / 2-s / 2}\right) \frac{d y}{y^{2}} .
\end{aligned}
$$

Here $f_{0}(y)$ is the constant term of $f$. The second term in the last equation is just a one-dimensional transform, hence of rapid decrease in $s$. For the first, we apply the Lemma and derive the Conjecture with an explicit value $r=1 / 2$. 
(2) Here I recall one of the standard ways (explained most clearly in[Colin de Verdière:1983]) to construct Eisenstein series in at least a part of the region outside of where the series converge.

Let $\rho(y)$ be 1 for $y \geq Y+1,0$ for $y \leq Y$.

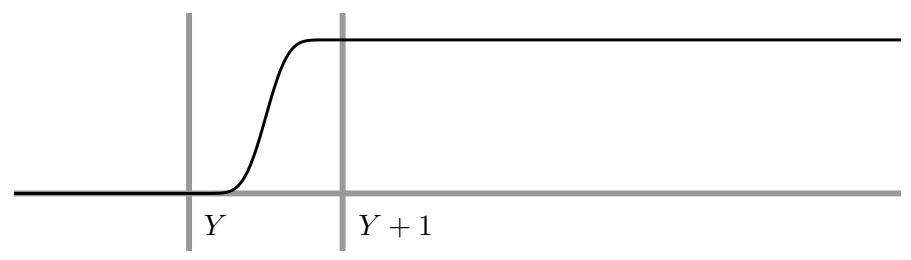

Figure 2. The smooth truncation function $\rho(y)$

We may identify $\rho(y) y^{s}$ with a function $E_{1, s}(y)$ on $\Gamma \backslash \mathcal{H}$. Let $E_{0, s}=E_{s}-E_{1, s}$, $\lambda_{s}=\left(s^{2} / 4-1 / 4\right)$. The function $E_{1, s}$ is defined for all $s$, a nd $E_{0, s}$, which is square-integrable, is defined at first only for $\operatorname{RE}(s)>1$. Since $\Delta E_{s}=\lambda_{s} E_{s}$

$$
\left(\Delta-\lambda_{s}\right) E_{0, s}=-\left(\Delta-\lambda_{s}\right) E_{1, s}=\text { (say) } F_{s} .
$$

The function $F_{s}$ has compact support on $\Gamma \backslash \mathcal{H}$, and is defined for all $s$. But now whenever $\operatorname{RE}(s)>0$ and $s$ does not lie in the real interval $(0,1], \lambda_{s}$ will be in the resolvent set of $\Delta$, so that $\left(\Delta-\lambda_{s}\right)^{-1} F_{s}$ is a well defined smooth function in $\mathrm{L}^{2}(\Gamma \backslash \mathcal{H})$ that agrees with $E_{0, s}$ for $\operatorname{RE}(s)>1$. I'll continue to call it $E_{0, s}$. The definition of $E_{s}$ may thus be continued into this region if we set

$$
E_{s}=E_{0, s}+E_{1, s}
$$

Thus we have an analytic continuation of $E_{s}$ to that region as well. Furthermore, the spectral theorem allows us to deduce an $\mathrm{L}^{2}$-norm for $E_{1, s}$ in terms of one of $F_{s}$, and since

$$
\begin{aligned}
\Delta \rho(y) y^{r} & =y^{2} \frac{\partial^{2} \rho(y) y^{r}}{\partial y^{2}} \\
& =\rho^{\prime \prime}(y) y^{r+2}+2 r \rho^{\prime}(y) y^{r+1}+r(r-1) \rho(y) y^{r} \\
(\Delta-r(r-1)) \rho(y) y^{r} & =\rho^{\prime \prime}(y) y^{r+2}+2 r \rho^{\prime}(y) y^{r+1}
\end{aligned}
$$

the $\mathrm{L}^{2}$-norm of $F_{s}$ is $O(|s|)$ when $\mathrm{Re}(s)$ is bounded. As in the first argument, this finishes off the proof of the Conjecture in this case.

The first argument uses the full standard truncation, the second only a partial and smooth one. The first argument will go through with little modification for Eisenstein series associated to cusp forms for a maximal proper rational parabolic subgroup, and Arthur's analogue of the Maass-Selberg formula for Eisenstein series associated to cusp forms will allow the Conjecture to be verified for that case. 
The second argument was applied to the same Eisenstein series in [Müller: 1989], who also extended Colin de Verdière's proof of the full meromorphic continuation of Eisenstein series to that case. The virtue of this argument is that useful partial truncations can be applied to Eisenstein series associated to arbitrary squareintegrable forms. I'll say something more about this later on. In any case, the one basic idea that both arguments illustrate is that of applying some kind of truncation to an Eisenstein series and looking at the pairing of $f$ in $\mathfrak{S}(\Gamma \backslash G)$ with each component separately.

\section{UnRAMified EISENSTEIN SERIES}

From now on, I'll assume that $G$ is split over $\mathbb{Q}$ and that $\Gamma$ is the full integral group $G(\mathbb{Z})$. This will simplify things a lot, and in inessential ways. My only aim is to explain a few basic ideas, after all.

Fix a Borel subgroup $P_{\emptyset}=A_{\emptyset} N_{\emptyset}$ in $G$. Set

$$
\begin{array}{ll}
\Delta & \text { the corresponding set of simple roots } \\
\Sigma & \text { the complete set of roots } \\
\Sigma^{+} & \text {the positive roots } \\
W & \text { the associated Weyl group }
\end{array}
$$

For every $\Theta \subset \Delta$, let

$P_{\Theta}=M_{\Theta} N_{\Theta}$ the corresponding standard parabolic subgroup containing $P_{\emptyset}$ $\Sigma^{+}(\Theta) \quad$ the linear span of $\Theta$ in $\Sigma^{+}$

Under my assumption on $\Gamma$, every rational parabolic subgroup of $G$ is conjugate to a unique $P_{\Theta}$.

An unramified character of $A$ is an analytic homomorphism from $A$ to $\mathbb{C}^{\times}$that's trivial on $K \cap A$. An unramified character of $P=P_{\emptyset}$ is one that factors through an unramified character of $A$ and the canonical surjection $P \rightarrow A$. If $\chi$ is an unramified character of $A_{P}$ and $\lambda$ is in $X_{*}(A)=\operatorname{Hom}_{\mathrm{alg}}\left(\mathbb{R}^{\times}, A\right)$, then

$$
\chi(\lambda(x))=|x|^{s_{\lambda}}
$$

for a uniquely determined complex number $s_{\lambda}$. This allows us to identify the space of unramified analytic characters of $P$ with elements in the complex vector space

$$
X_{\mathbb{C}}^{*}(A)=\operatorname{Hom}\left(X_{*}(A), \mathbb{C}\right) .
$$

In these terms, for $s$ in $X_{\mathbb{C}}^{*}(A)$

$$
s_{\lambda}=\left\langle s, \lambda^{\vee}\right\rangle \text {. }
$$


The lattice of weights $X^{*}(A)=\operatorname{Hom}\left(X_{*}(A), \mathbb{Z}\right)$ embeds canonically into this space. The space of unramified characters $X_{\Theta}^{*}$ of $P_{\Theta}$ may be identified with those linear functions in $X_{\mathbb{C}}^{*}(A)$ that are trivial on the coroots $\alpha^{\vee}$ for $\alpha$ in $\Theta$. The cone $X_{\Theta}^{++}$is the subset of $X_{\Theta}^{*}$ where $s_{\alpha}>0$ for $\alpha$ in the complement of $\Theta$.

From now on, I'll work only with functions on $G$ fixed on the right by $K$-in effect functions on the symmetric space $G / K$.

As I have already remarked, if $\chi$ is an unramified character of $P_{\emptyset}$ it extends to a unique function on all of $G$ right-invariant under $K$ and left-invariant under $N_{\emptyset}\left(\Gamma \cap P_{\emptyset}\right)$. If $\chi=\chi_{s}$ then the associated Eisenstein series is the function

$$
E_{s}(g)=\sum_{\Gamma \cap P_{\emptyset} \backslash \Gamma} \chi_{s}(\gamma g) \delta_{\emptyset}^{1 / 2}(\gamma g) .
$$

on $\Gamma \backslash G / K$. This converges whenever

$$
\operatorname{RE}\left(s_{\alpha}\right)>1
$$

for all $\alpha$ in $\Delta$, and is then an automorphic form. The function $E_{s}$ continues meromorphically to all $s$, with a simple pole along each of the affine subsets $s_{\alpha}=1(\alpha$ in $\Delta)$, as well as lots of other singularities. If $G$ is semi-simple, this intersection is the single point $\chi=\delta_{\emptyset}^{1 / 2}$ and the residue of the Eisenstein series at that point, which is just a constant function in this case, is known from Langlands' work on the Tamagawa number to be the inverse of the volume of the quotient $\Gamma \backslash G$. More generally, the Laurent expansions at singularities of the Eisenstein series give rise to automorphic forms on $\Gamma \backslash G$. Some of these forms are squareintegrable modulo the centre of $G$, as is the residue for $\delta_{\emptyset}^{1 / 2}$. The principal result of Langlands' work on Eisenstein series is that the entire $\mathrm{L}^{2}$-spectrum of $\Gamma \backslash G$ is constructed from the Eisenstein series associated to cusp forms on parabolic quotients, residues of these, and Eisenstein series built up in turn from such residues.

The Eisenstein series construction is transitive, as is the construction of representations induced from parabolic subgroups. I'll be interested in just one case of this. On the one hand, consider the constant functions of $M_{\Theta}$, which are generated by the residue of the Eisenstein series associated to the image of $P_{\emptyset}$ in $M_{\Theta}$ for the character $\delta_{\emptyset}^{1 / 2} \delta_{\Theta}^{-1 / 2}$ of $A_{\emptyset}$. Let $\rho_{\Theta}$ be the corresponding element of $X_{\mathbb{C}}(P)$. It is characterized by these properties: (a) $\rho_{\Theta}$ lies in the linear span of $\Delta-\Theta$; (b) $\left\langle\rho_{\Theta}, \alpha^{\vee}\right\rangle=1$ for $\alpha$ in $\Theta$. This residue is also the Eisenstein series associated to $P_{\Theta}$ and $\rho_{\Theta}$ on $G$. Thus

The residue of the Eisenstein series on $G$ associated to $P_{\emptyset}$ along the whole affine subspace where $s_{\alpha}=1$ for $\alpha$ in $\Theta$ agrees with the residual Eisenstein series induced by the space of constant functions from $P_{\Theta}$ to $G$. 
I now want to explore more precisely what my Conjecture means for these residual Eisenstein series.

If $\Phi$ is any function on $\Gamma \backslash G$, its constant term with respect to the rational parabolic subgroup $P$ is the function

$$
\Pi_{P} \Phi(g)=\int_{\Gamma \cap N_{P} \backslash N_{P}} \Phi(n g) d n
$$

on $N_{P}(\Gamma \cap P) \backslash G$. Since $G=P K$, the symmetric space $G / K$ may be identified with $P / K \cap P$ and since $N \backslash P=M$ this quotient may in turn be identified with $\Gamma_{M} \backslash M / K_{M}$ where $K_{M}$ is the image of $K \cap P$ in $M$. Thus the constant term of an automorphic form on $\Gamma \backslash G / K$ may be identified with an automorphic form on $\Gamma_{M} \backslash M / K_{M}$.

The map from $\Phi$ to $\Pi_{P} \Phi$ is in some sense adjoint to the Eisenstein series construction from functions on $N_{P}(\Gamma \cap P) \backslash G$ to those on $\Gamma \backslash G$. For us there is one practical meaning to this. If $f$ lies in the Schwartz space of $N(\Gamma \cap P) \backslash G$ then the 'pseudo-Eisenstein series'

$$
E_{f}(g)=\sum_{\Gamma \cap P \backslash \Gamma} f(\gamma g)
$$

defines a function in the Schwartz space of $G$. The adjoint relation between Eisenstein series and constant term means that

$$
\left\langle E_{f}, E_{\varphi}\right\rangle=\int_{N(\Gamma \cap P) \backslash G} f(x) E_{\varphi, P} d g
$$

This also says that an Eisenstein series is determined as a distribution by its constant term, since the image of pseudo-Eisenstein series from all rational parabolic subgroups is dense in the Schwartz space.

The constant term of $E_{s}$ for generic $s$ with respect to $P_{\emptyset}$ is

$$
\delta^{1 / 2}(g) \sum_{W} w \chi_{s}(g) \prod_{\lambda>0, w \lambda<0} \frac{\xi\left(s_{\lambda}\right)}{\xi\left(s_{\lambda}+1\right)}=\delta^{1 / 2}(g) \sum_{W} w \chi_{s}(g) \prod_{\lambda>0, w \lambda<0} \Xi\left(s_{\lambda}\right)
$$

Recall that $s_{\lambda}=\left\langle s, \lambda^{\vee}\right\rangle$. The constant term of a residue is the residue of the 
constant term. Thus, for example, when $\chi=\delta_{\emptyset}^{1 / 2}$ and $\sigma=s_{\chi}$ the residues of all the terms in this sum vanish except for $w=w_{\ell}$, the longest element in $W$, and then we get

$$
\frac{\prod_{\lambda>0, \lambda \notin \Delta} \xi\left(\sigma_{\lambda}\right)}{\prod_{\lambda>0} \xi\left(\sigma_{\lambda}+1\right)}
$$

One of the ingredients in Langlands' identification of the Tamagawa number is a well known theorem of[Kostant: 1959] that asserts some curious cancellations to take place in this quotient to make it

$$
\frac{1}{\prod_{1}^{r} \xi\left(a_{i}\right)}
$$

where $r=$ card $\Delta$ and the $a_{i}$ are the degrees of the polynomial ring of invariants of $W$ in $\mathbb{R}^{r}$. This has subsequently been proven by many means (see for example $\S 3.20$ of [Humphries: 1990]), but Kostant's original proof is still of interest.

\section{3. $\mathrm{SL}_{3}(\mathbb{R})$}

Because pseudo-Eisenstein series are in the Schwartz space, the Conjecture requires that the constant term of residues of Eisenstein series satisfy a certain growth condition in the positive cone $X^{++}(P)$. In this section and the next, I shall verify and comment on this in the cases where $G$ has rational rank two. The results themselves are also implied by the principal result of [Franke: 1999], but there is nonetheless some point in bringing them up here. The growth condition amounts to a combinatorial cancellation of terms in the numerators and denominators in the constant term, generalizations of Kostant's cancellation. These are of independent interest, since they are presumably closely related to conjectures of Arthur's about the discrete spectrum of $\mathrm{L}^{2}(\Gamma \backslash G)$. Such things have been observed before in, for example, work of Moeglin and Waldspurger, but I am not aware that the general phenomenon has been explained completely satisfactorily nor even fully recognized.

Now let $G=\mathrm{SL}_{3}(\mathbb{R}), \Gamma=\mathrm{SL}_{3}(\mathbb{Z})$. The group $A_{\emptyset}$ can be taken to be the group of diagonal matrices of determinant $1, P_{\emptyset}$ the subgroup of upper triangular matrices. Here $W$ has six elements, and there are three positive roots $\alpha, \beta$, and $\alpha+\beta$. Suppose that the root $\alpha$ takes $\left(a_{i}\right)$ to $a_{1} / a_{2}, \beta$ takes it to $a_{2} / a_{3}$. Then

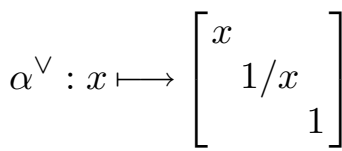

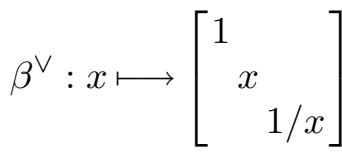




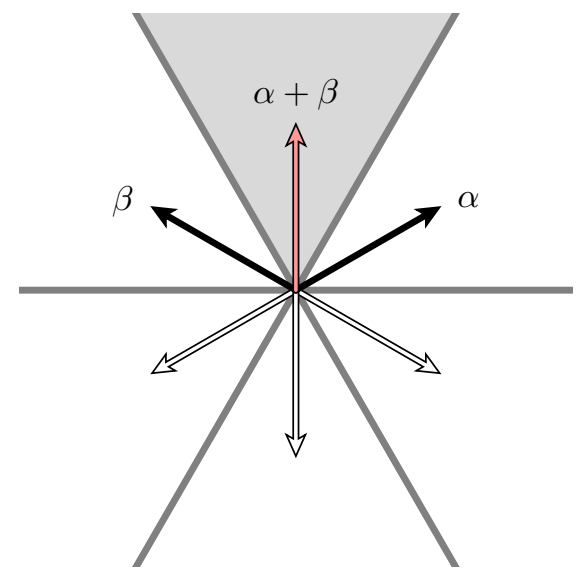

Figure 3. The root system for $\mathrm{SL}_{3}$

How the Weyl group acts can be pictured nicely.

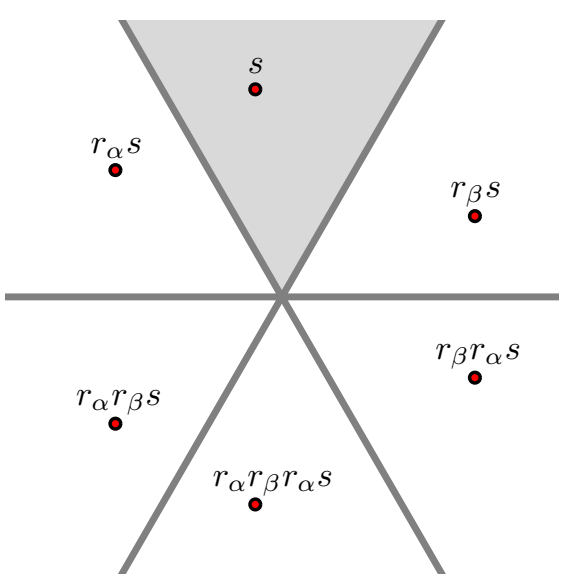

Figure 4. The Weyl group action for $\mathrm{SL}_{3}$

For each $w$ in $W$ let $R_{w}$ be the set of positive roots $\lambda$ such that $w \lambda<0$. If $\ell(x y)=\ell(x)+\ell(y)$ we have $R_{x y}=y^{-1} R_{x} \cup R_{y}$. Since $(\alpha+\beta)^{\vee}=\alpha^{\vee}+\beta^{\vee}$, the expansion of the constant term is given in this table: 


$\begin{array}{lll}w & R_{w} & \text { coefficient of } w \chi_{s} \\ 1 & \emptyset & 1 \\ r_{\alpha} & \alpha & \frac{\xi\left(s_{\alpha}\right)}{\xi\left(s_{\alpha}+1\right)} \\ r_{\beta} & \beta & \frac{\xi\left(s_{\beta}\right)}{\xi\left(s_{\beta}+1\right)} \\ r_{\alpha} r_{\beta} & \beta, \alpha+\beta & \frac{\xi\left(s_{\beta}\right)}{\xi\left(s_{\beta}+1\right)} \frac{\xi\left(s_{\alpha}+s_{\beta}\right)}{\xi\left(s_{\alpha}+s_{\beta}+1\right)} \\ r_{\alpha} r_{\beta} & \beta, \alpha+\beta & \frac{\xi\left(s_{\alpha}\right)}{\xi\left(s_{\alpha}+1\right)} \frac{\xi\left(s_{\alpha}+s_{\beta}\right)}{\xi\left(s_{\alpha}+s_{\beta}+1\right)} \\ r_{\alpha} r_{\beta} r_{\alpha}=r_{\beta} r_{\alpha} r_{\beta} & \alpha, \beta, \alpha+\beta & \frac{\xi\left(s_{\alpha}\right)}{\xi\left(s_{\alpha}+1\right)} \frac{\xi\left(s_{\beta}\right)}{\xi\left(s_{\beta}+1\right)} \frac{\xi\left(s_{\alpha}+s_{\beta}\right)}{\xi\left(s_{\alpha}+s_{\beta}+1\right)}\end{array}$

The table can be summarized in a diagram.

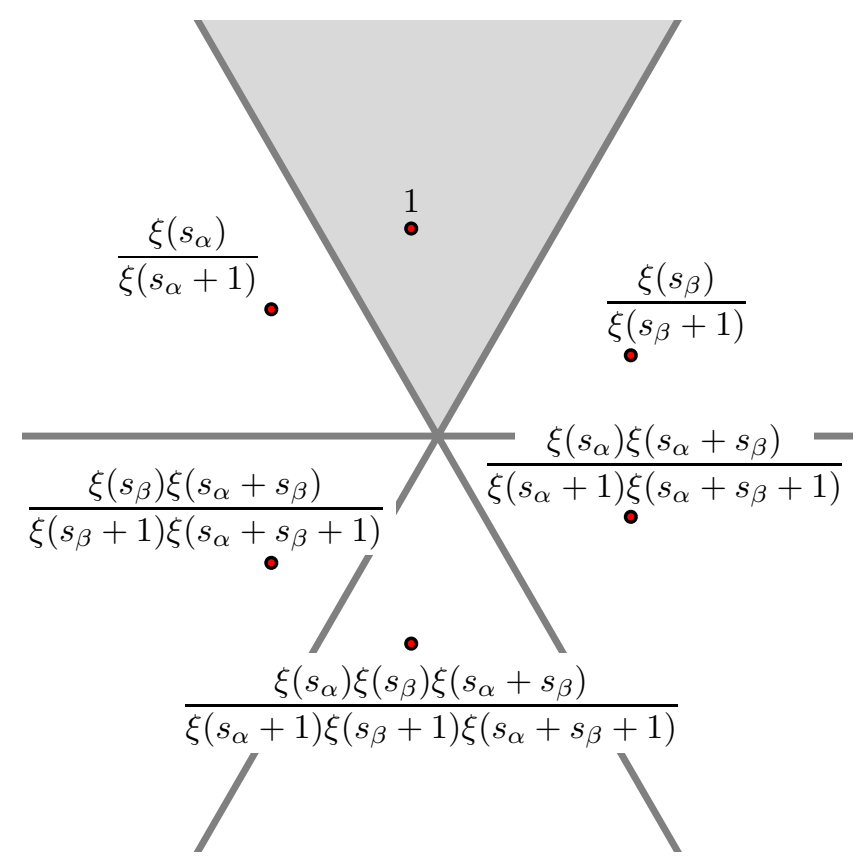

Figure 5. Coefficients for the constant term of $E_{s}$

As I have already remarked, the behaviour of an Eisenstein series is essentially determined by that of its constant term. The constant term in the case at hand has poles where the numerator $\xi\left(s_{\alpha}\right) \xi\left(s_{\beta}\right) \xi\left(s_{\alpha}+s_{\beta}\right)$ does, but also where the denominator

$$
\xi\left(s_{\alpha}+1\right) \xi\left(s_{\beta}+1\right) \xi\left(s_{\alpha}+s_{\beta}+1\right)
$$

has zeroes. You can anticipate the problem - conjecturally we know some very severe restrictions on the location of these zeroes, but in practice we can only say that they are located in the regions $-1<\operatorname{RE}\left(s_{\alpha}\right)<0,-1<\operatorname{RE}\left(s_{\beta}\right)<0$, $-1<\operatorname{RE}\left(s_{\alpha}+s_{\alpha}\right)<0$. The general idea is that Eisenstein series behave badly as 
we exit the positive cone. The Eisenstein series induced from $P_{\emptyset}$ really do behave badly, too. Among other things, we can see directly now that the two-dimensional residue of the constant term of $E_{s}$ at $s=\rho_{\Delta}$ is the constant function

$$
\frac{\xi(2)}{\xi(2) \xi(2) \xi(3)}=\frac{1}{\xi(2) \xi(3)}
$$

The Eisenstein series and its constant term also have simple poles along the lines $s_{\alpha}=1$ and $s_{\beta}=1$. Since these are equivalent under automorphism, we may just look at one, say the first. The residue of the constant term is the sum of the residues of the individual terms. The residue vanishes unless $w \alpha<0$. Thus this table gives the constant terms along $s_{\alpha}=1$ :

$$
\begin{array}{lll}
w & R_{w} & \text { coefficient of } w \chi_{s} \\
r_{\alpha} & \alpha & \frac{1}{\xi(2)} \\
r_{\beta} r_{\alpha} & \alpha, \alpha+\beta & \frac{1}{\xi(2)} \frac{\xi\left(s_{\beta}\right)}{\xi\left(s_{\beta}+1\right)} \\
r_{\alpha} r_{\beta} r_{\alpha}=r_{\beta} r_{\alpha} r_{\beta} & \alpha, \beta, \alpha+\beta & \frac{1}{\xi(2)} \frac{\xi\left(s_{\beta}\right)}{\xi\left(s_{\beta}+1\right)} \frac{\xi\left(s_{\beta}+1\right)}{\xi\left(s_{\beta}+2\right)}
\end{array}
$$

From now on I am going to leave out the constant $\xi(2)$ in the denominator, which amounts to normalizing the Eisenstein series by a factor of $\xi(2)$.

It is the last coefficient which interests us, since (taking into account my normalization) it can be simplified:

$$
\frac{\xi\left(s_{\beta}\right)}{\xi\left(s_{\beta}+1\right)} \frac{\xi\left(s_{\beta}+1\right)}{\xi\left(s_{\beta}+2\right)}=\frac{\xi\left(s_{\beta}\right)}{\xi\left(s_{\beta}+2\right)} .
$$

This cancellation is in fact required by the Conjecture. It is also required, incidentally, by the principal Theorem of [Franke: 1992], which is that all the poles in the positive cone must be real.

According to what I have said earlier, these representations make up the residue of the Eisenstein series $E_{s}$ on the complex line $s_{\alpha}=1$. This is because the Eisenstein series constructed from $P_{\emptyset}$ to $G$ can be built in two stages, first from $P_{\emptyset}$ to $P_{\{\alpha\}}$ and then from $P_{\{\alpha\}}$ to $G$. The first is an extension of that from $P_{\emptyset} \cap M$ to $M$, and the character $\chi_{s}$ is a residue of that along $s_{\alpha}=1$. 


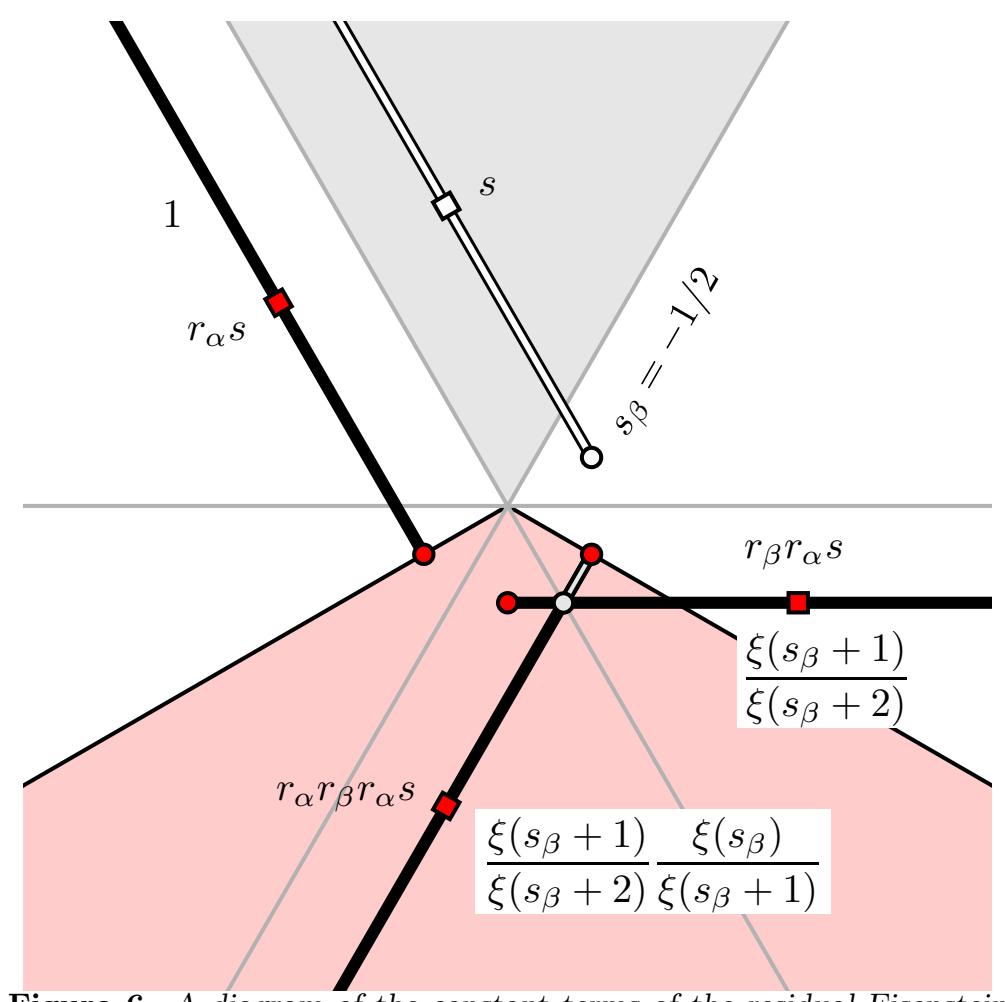

Figure 6. A diagram of the constant terms of the residual Eisenstein series. The endpoints of the rays mark the real part of the $\mathrm{L}^{2}$-spectrum. The coordinate $s_{\beta}$ is that of the original point at which the residue is taken, so the term with $\xi\left(s_{\beta}+1\right)$ in its denominator has a potential problem when the ray exits the positive chamber. The obtuse cone at the bottom is the region in which constant terms are square-integrable. The bar 0 on the one ray marks the region $s_{\beta}<0$ of potential trouble.

What's going on here? Suppose again, for the moment, that $G$ is an arbitrary split group, $P=P_{\Theta}$, and that we are looking at the residue along the subspace $s_{\alpha}=1$ for $\alpha$ in $\Theta$. The constant term of the residue along the affine space $s_{\alpha}=1$ for $\alpha$ in $\Theta$ is

$$
\delta_{\Theta}^{1 / 2} \sum_{w \Theta<0} w \chi_{s}\left(\frac{\prod_{\lambda>0, w \lambda<0, \lambda \notin \Theta} \xi\left(s_{\lambda}\right)}{\prod_{\lambda>0, w \lambda<0} \xi\left(s_{\lambda}+1\right)}\right) .
$$

Here, if $\lambda^{\vee}=\sum_{\Theta} n_{\alpha} \alpha^{\vee}+\sum_{\Delta-\Theta} n_{\alpha} \alpha^{\vee}$ then

$$
\left\langle s, \lambda^{\vee}\right\rangle=\sum_{\Theta} m_{\alpha}+\sum_{\Delta-\Theta} n_{\alpha} s_{\alpha} .
$$


If $\lambda$ lies in $\Sigma^{+}(\Theta)$ then these terms are constants, and we can normalize the Eisenstein series so as to get rid of them. The constant term then becomes

$$
\delta_{\Theta}^{1 / 2} \sum_{w \Theta<0} w \chi_{s} \prod_{\substack{\lambda>0, w \lambda<0 \\ \lambda \notin \Sigma^{+}(\Theta)}} \frac{\xi\left(s_{\lambda}\right)}{\xi\left(s_{\lambda}+1\right)} .
$$

The denominator $\xi\left(s_{\lambda}+1\right)$ will cause trouble if $\left\langle\rho_{\Theta}, \lambda^{\vee}\right\rangle<0$, and then in that case, according to the Conjecture (or according to [Franke:1989]) it must be canceled out by a term in the numerator.

We have therefore this combinatorial result:

Suppose $w \Theta<0$. The expressions $s_{\lambda}+1$ with (a) $\lambda>0$, (b) $w \lambda<0$, $(c) \lambda \notin \Sigma^{+}(\Theta)$ and $(d)\left\langle\rho_{\Theta}, \lambda^{\vee}\right\rangle<0$ are matched by expressions $s_{\mu}$ with $\mu$ satisfying (a)-(c).

This is intriguingly related to Kostant's cancellation theorem, and it ought not to be too hard to prove directly. Of course it is only the simplest case of a much more general conjecture that applies to residues more complicated than the constant functions on the rational parabolic subgroups. But since in general we know so little about the coefficients that appear in constant terms, we must find a way to understand the situation in a different way. There is in fact an interesting geometric interpretation of these cancellations that we shall come back to in the last section.

\section{OTHER RANK TWO GROUPS}

The situation for the other semi-simple groups of rank two can be deduced from tables and diagrams.

- The root system $C_{2}$

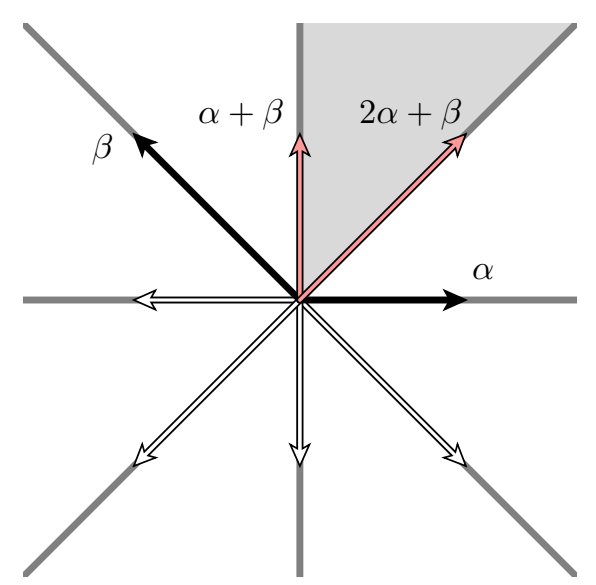

Figure 7. The root system $C_{2}$ 
The coroots are $\alpha^{\vee}, \beta^{\vee}, \alpha^{\vee}+\beta^{\vee}, \alpha^{\vee}+2 \beta^{\vee}$.

$\begin{array}{ll}w & \left.R_{w}^{\vee} \text { (coroots associated to } R_{w}\right) \\ 1 & \emptyset \\ r_{\alpha} & \alpha^{\vee} \\ r_{\beta} & \beta^{\vee} \\ r_{\beta} r_{\alpha} & \alpha^{\vee}, \alpha^{\vee}+\beta^{\vee} \\ r_{\alpha} r_{\beta} & \beta^{\vee}, \alpha^{\vee}+2 \beta^{\vee} \\ r_{\alpha} r_{\beta} r_{\alpha} & \alpha^{\vee}, \alpha^{\vee}+\beta^{\vee}, \alpha^{\vee}+2 \beta^{\vee} \\ r_{\beta} r_{\alpha} r_{\beta} & \beta^{\vee}, \alpha^{\vee}+2 \beta^{\vee}, \alpha^{\vee}+\beta^{\vee} \\ r_{\beta} r_{\alpha} r_{\beta} r_{\alpha}=r_{\alpha} r_{\beta} r_{\alpha} r_{\beta} & \alpha^{\vee}, \alpha^{\vee}+\beta^{\vee}, \alpha^{\vee}+2 \beta^{\vee}, \beta^{\vee}\end{array}$

Coefficients for the residue along $s_{\alpha}=1$ :

$\begin{array}{ll}w & \text { coefficient of } w \chi_{s} \\ r_{\alpha} & 1 \\ r_{\beta} r_{\alpha} & \frac{\xi\left(s_{\beta}+1\right)}{\xi\left(s_{\beta}+2\right)} \\ r_{\alpha} r_{\beta} r_{\alpha} & \frac{\xi\left(s_{\beta}+1\right)}{\xi\left(s_{\beta}+2\right)} \frac{\xi\left(2 s_{\beta}+1\right)}{\xi\left(2 s_{\beta}+2\right)} \\ r_{\beta} r_{\alpha} r_{\beta} r_{\alpha} & \frac{\xi\left(s_{\beta}+1\right)}{\xi\left(s_{\beta}+2\right)} \frac{\xi\left(2 s_{\beta}+1\right)}{\xi\left(2 s_{\beta}+2\right)} \frac{\xi\left(s_{\beta}\right)}{\xi\left(s_{\beta}+1\right)}\end{array}$

Since $\left\langle\sigma_{\alpha}, \beta^{\vee}\right\rangle=-1 / 2$, the dangerous term is $\xi\left(s_{\beta}+1\right)$, which is indeed cancelled.

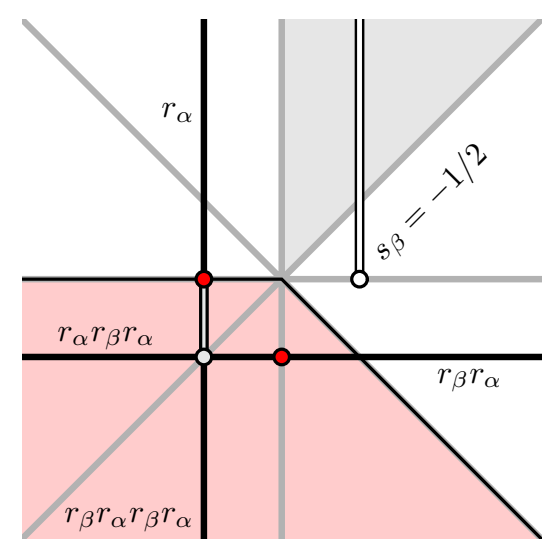

Figure 8. Constant terms for the residue along $s_{\alpha}=1$. The potentially singular region is marked by a bar.

Coefficients for the residue along $s_{\beta}=1$ : 


$\begin{array}{ll}w & \text { coefficient of } w \chi_{s} \\ r_{\beta} & 1 \\ r_{\alpha} r_{\beta} & \frac{\xi\left(s_{\alpha}+2\right)}{\xi\left(s_{\alpha}+3\right)} \\ r_{\beta} r_{\alpha} r_{\beta} & \frac{\xi\left(s_{\alpha}+2\right)}{\xi\left(s_{\alpha}+3\right)} \frac{\xi\left(s_{\alpha}+1\right)}{\xi\left(s_{\alpha}+2\right)} \\ r_{\alpha} r_{\beta} r_{\alpha} r_{\beta} & \frac{\xi\left(s_{\alpha}+2\right)}{\xi\left(s_{\alpha}+3\right)} \frac{\xi\left(s_{\alpha}+1\right)}{\xi\left(s_{\alpha}+2\right)} \frac{\xi\left(s_{\alpha}\right)}{\xi\left(s_{\alpha}+1\right)}\end{array}$

Since $\left\langle\sigma_{\beta}, \alpha^{\vee}\right\rangle=-1$, the dangerous term is again $\xi\left(s_{\alpha}+1\right)$, which is cancelled.

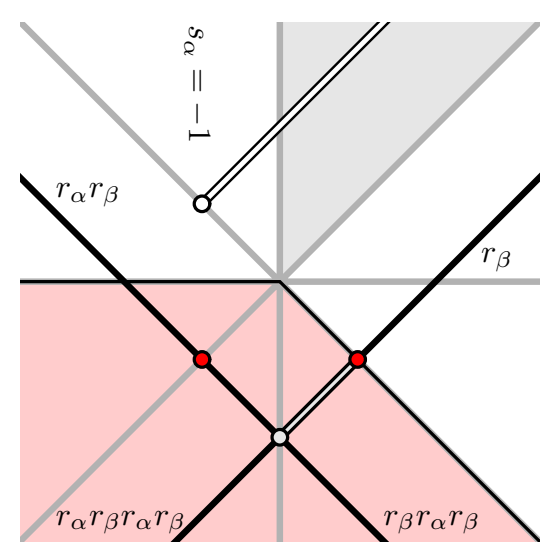

Figure 9. Constant term for the residue along $s_{\beta}=1$

- The root system $G_{2}$

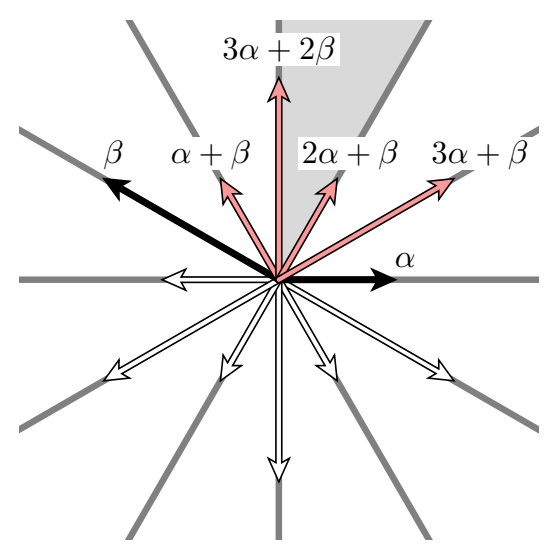

Figure 10. The root system $G_{2}$

The coroots are $\alpha^{\vee}, \beta^{\vee}, \alpha^{\vee}+\beta^{\vee}, \alpha^{\vee}+2 \beta^{\vee}, \alpha^{\vee}+3 \beta^{\vee}, 2 \alpha^{\vee}+3 \beta^{\vee}$. 


$\begin{array}{ll}w & R_{w}^{\vee} \\ 1 & \emptyset \\ r_{\alpha} & \alpha^{\vee} \\ r_{\beta} & \beta^{\vee} \\ r_{\beta} r_{\alpha} & \alpha^{\vee}, \alpha^{\vee}+\beta^{\vee} \\ r_{\alpha} r_{\beta} & \beta^{\vee}, \alpha^{\vee}+3 \beta^{\vee} \\ r_{\alpha} r_{\beta} r_{\alpha} & \alpha^{\vee}, \alpha^{\vee}+\beta^{\vee}, 2 \alpha^{\vee}+3 \beta^{\vee} \\ r_{\beta} r_{\alpha} r_{\beta} & \beta^{\vee}, \alpha^{\vee}+3 \beta^{\vee}, \alpha^{\vee}+2 \beta^{\vee} \\ r_{\beta} r_{\alpha} r_{\beta} r_{\alpha} & \alpha^{\vee}, \alpha^{\vee}+\beta^{\vee}, 2 \alpha^{\vee}+3 \beta^{\vee}, \alpha^{\vee}+2 \beta^{\vee} \\ r_{\alpha} r_{\beta} r_{\alpha} r_{\beta} & \beta^{\vee}, \alpha^{\vee}+3 \beta^{\vee}, \alpha^{\vee}+2 \beta^{\vee}, 2 \alpha^{\vee}+3 \beta^{\vee} \\ r_{\alpha} r_{\beta} r_{\alpha} r_{\beta} r_{\alpha} & \alpha^{\vee}, \alpha^{\vee}+\beta^{\vee}, 2 \alpha^{\vee}+3 \beta^{\vee}, \alpha^{\vee}+2 \beta^{\vee}, \alpha^{\vee}+3 \beta^{\vee} \\ r_{\beta} r_{\alpha} r_{\beta} r_{\alpha} r_{\beta} & \beta^{\vee}, \alpha^{\vee}+3 \beta^{\vee}, \alpha^{\vee}+2 \beta^{\vee}, 2 \alpha^{\vee}+3 \beta^{\vee}, \alpha^{\vee}+\beta^{\vee} \\ r_{\beta} r_{\alpha} r_{\beta} r_{\alpha} r_{\beta} r_{\alpha}=r_{\alpha} r_{\beta} r_{\alpha} r_{\beta} r_{\alpha} r_{\beta} & \beta^{\vee}, \alpha^{\vee}+3 \beta^{\vee}, \alpha^{\vee}+2 \beta^{\vee}, 2 \alpha^{\vee}+3 \beta^{\vee}, \alpha^{\vee}+\beta^{\vee}, \alpha^{\vee}\end{array}$

Coefficients for the residue along $s_{\alpha}=1$ :

$\begin{array}{ll}w & \text { coefficient of } w \chi_{s} \\ r_{\alpha} & 1 \\ r_{\beta} r_{\alpha} & \frac{\xi\left(s_{\beta}+1\right)}{\xi\left(s_{\beta}+2\right)} \\ r_{\alpha} r_{\beta} r_{\alpha} & \frac{\xi\left(s_{\beta}+1\right)}{\xi\left(s_{\beta}+2\right)} \frac{\xi\left(3 s_{\beta}+2\right)}{\xi\left(3 s_{\beta}+3\right)} \\ r_{\beta} r_{\alpha} r_{\beta} r_{\alpha} & \frac{\xi\left(s_{\beta}+1\right)}{\xi\left(s_{\beta}+2\right)} \frac{\xi\left(3 s_{\beta}+2\right)}{\xi\left(3 s_{\beta}+3\right)} \frac{\xi\left(2 s_{\beta}+1\right)}{\xi\left(2 s_{\beta}+2\right)} \\ r_{\alpha} r_{\beta} r_{\alpha} r_{\beta} r_{\alpha} & \frac{\xi\left(s_{\beta}+1\right)}{\xi\left(s_{\beta}+2\right)} \frac{\xi\left(3 s_{\beta}+2\right)}{\xi\left(3 s_{\beta}+3\right)} \frac{\xi\left(2 s_{\beta}+1\right)}{\xi\left(2 s_{\beta}+2\right)} \frac{\xi\left(3 s_{\beta}+1\right)}{\xi\left(3 s_{\beta}+2\right)} \\ r_{\beta} r_{\alpha} r_{\beta} r_{\alpha} r_{\beta} r_{\alpha} & \frac{\xi\left(s_{\beta}+1\right)}{\xi\left(s_{\beta}+2\right)} \frac{\xi\left(3 s_{\beta}+2\right)}{\xi\left(3 s_{\beta}+3\right)} \frac{\xi\left(2 s_{\beta}+1\right)}{\xi\left(2 s_{\beta}+2\right)} \frac{\xi\left(3 s_{\beta}+1\right)}{\xi\left(3 s_{\beta}+2\right)} \frac{\xi\left(s_{\beta}\right)}{\xi\left(s_{\beta}+1\right)}\end{array}$

Since $\left\langle\sigma_{\alpha}, \beta^{\vee}\right\rangle=-1 / 2$, the terms $s_{\beta}+1$ and $3 s_{\beta}+2$ ought to be-and arecancelled.

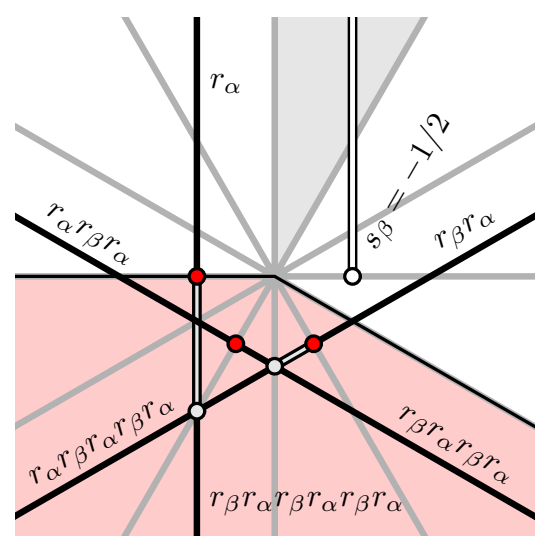

Figure 11. Constant term for the residue along $s_{\alpha}=1$ 
Coefficients for the residue along $s_{\beta}=1$ :

$w$

$r_{\beta}$

$r_{\alpha} r_{\beta}$

$r_{\beta} r_{\alpha} r_{\beta}$

$r_{\alpha} r_{\beta} r_{\alpha} r_{\beta}$

$r_{\beta} r_{\alpha} r_{\beta} r_{\alpha} r_{\beta}$

$r_{\alpha} r_{\beta} r_{\alpha} r_{\beta} r_{\alpha} r_{\beta}$ coefficient of $w \chi_{s}$

1

$\frac{\xi\left(s_{\alpha}+3\right)}{\xi\left(s_{\alpha}+4\right)}$

$\frac{\xi\left(s_{\alpha}+3\right)}{\xi\left(s_{\alpha}+4\right)} \frac{\xi\left(s_{\alpha}+2\right)}{\xi\left(s_{\alpha}+3\right)}$

$\frac{\xi\left(s_{\alpha}+3\right)}{\xi\left(s_{\alpha}+4\right)} \frac{\xi\left(s_{\alpha}+2\right)}{\xi\left(s_{\alpha}+3\right)} \frac{\xi\left(2 s_{\alpha}+3\right)}{\xi\left(2 s_{\alpha}+4\right)}$

$\frac{\xi\left(s_{\alpha}+3\right)}{\xi\left(s_{\alpha}+4\right)} \frac{\xi\left(s_{\alpha}+2\right)}{\xi\left(s_{\alpha}+3\right)} \frac{\xi\left(2 s_{\alpha}+3\right)}{\xi\left(2 s_{\alpha}+4\right)} \frac{\xi\left(s_{\alpha}+1\right)}{\xi\left(s_{\alpha}+2\right)}$

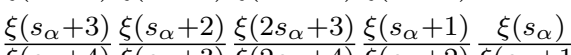

$\frac{\xi\left(s_{\alpha}+4\right)}{\xi\left(s_{\alpha}+3\right)} \overline{\xi\left(2 s_{\alpha}+4\right)} \frac{\xi\left(s_{\alpha}+2\right)}{\xi\left(s_{\alpha}+1\right)}$

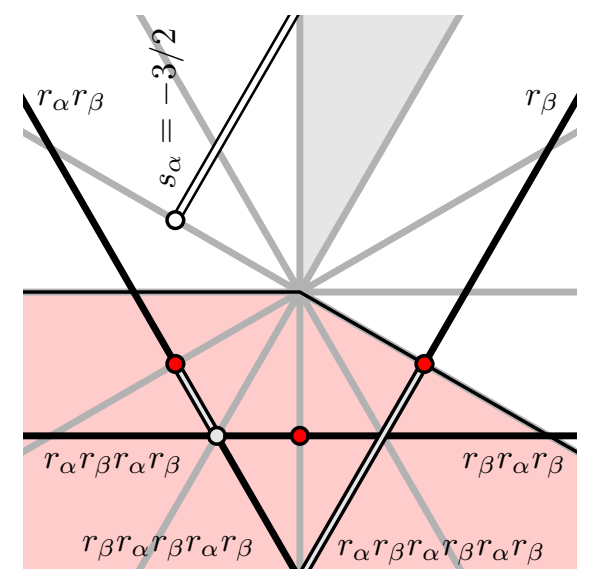

Figure 12. Constant term for the residue along $s_{\beta}=1$

Here $\left\langle\alpha, \beta^{\vee}\right\rangle=-3 / 2$. The dangerous range is $s_{\alpha}=0$ to $-3 / 2$. The dangerous terms are $s_{\alpha}+1$ and $s_{\alpha}+2$, which are cancelled.

I have marked by bars $=0$ in all these diagrams the regions on the spectrum of the constant terms where singularities would occur if cancellation didn't occur. As I shall explain in the next section, it is significant that these barred segments all lie inside the obtuse cone signifying square-integrability.

\section{How to Prove the CONJeCture?}

I have in mind a two-step process for proving the conjecture. The first is to prove it for Eisenstein series associated to maximal rational parabolic subgroups, the case in which the Eisenstein series form a one-parameter family. The second is to go on from this to higher rank situations. 
Both steps use Arthur's truncation operators. The second step looks almost straightforward - one can in principle find a formula for the inner product of truncations of arbitrary Eisenstein series, generalizing Arthur's formula for those associated to cusp forms. The coefficients in the formula will involve products of intertwining operators asssociated to maximal parabolic subgroups, so that if one has dealt with that case one should be in good shape - one can then use the last part of the first method of $\S 1$ to conclude. In this step, the hard part is finding an inner product formula explicit enough to work with. I am not so sure how difficult this will be, but it doesn't seem too bad. In particular, although I'll not do it here, one can use the cancellations observed earlier in this paper to prove the conjecture for all groups of rational rank two.

The more interesting step is the first. What I think will work for this is an extension of the technique explained in [Colin de Verdière:1983] to construct Eisenstein series for $\mathrm{SL}_{2}(\mathbb{Z})$. Here is the basic idea: suppose that $E$ is an automorphic form on $\mathfrak{X}=G / K$ that can be written as a sum $E_{0}+E_{1}$, with $E_{0}$ square-integrable. The $E_{i}$ in practice will be obtained by a kind of truncation, and will not be smooth. We can find $\varphi$ in $C^{\infty}(K \backslash G / K)$ (of arbitrarily small support) such that $R_{\varphi} E=E$. Then also

$$
E=R_{\varphi} E=R_{\varphi} E_{0}+R_{\varphi} E_{1} .
$$

The components $E_{0}$ and $E_{1}$ might not be smooth, but both $R_{\varphi} E_{0}$ and $R_{\varphi} E_{1}$ will be. The function $R_{\varphi} E_{0}$ will in fact lie in $\mathrm{L}^{2, \infty}(\Gamma \backslash \mathfrak{X})$. If $\Delta E=\lambda E$ then, since $R_{\varphi}$ and $\Delta$ commute,

$$
\begin{aligned}
0 & =(\Delta-\lambda) E \\
& =(\Delta-\lambda) R_{\varphi} E_{0}+(\Delta-\lambda) R_{\varphi} E_{1} \\
(\Delta-\lambda) R_{\varphi} E_{0} & =-(\Delta-\lambda) R_{\varphi} E_{1} \\
& =-R_{\varphi}(\Delta-\lambda) E_{1} \\
& =\text { (say) F. }
\end{aligned}
$$

Here $(\Delta-\lambda) E_{1}$ is a distribution. If $\lambda$ does not lie in the spectrum $\sigma_{\Delta}$ of $\Delta$,

$$
R_{\varphi} E_{0}=-(\Delta-\lambda)^{-1} F, \quad\left\|R_{\varphi} E_{0}\right\| \leq \frac{\|F\|}{\text { distance from } \lambda \text { to } \sigma_{\Delta}} .
$$

In the case that Colin de Verdière used this, $F$ has compact support and $\|F\|$ remains bounded near the interval $(0,1]$. The spectral theorem then implies that the poles on $(0,1]$ of $E_{s}$ as a function of $s$ are simple. However, for the group $G_{2}$ there is a well known example to be found in Appendix III of [Langlands:1976] of a case where the pole is not simple. Werner Müller in [Müller:1989] deduces from this that the method of Colin de Verdière cannot be applied in general to derive properties of Eisenstein series associated to square-integrable automorphic 
forms, but his deduction is fallacious. It is neither to be expected, nor necessary, nor even desired that $F$ have compact support or that $\|F\|$ remain bounded near the real $s$-axis. One can get by with a lot less. The important point is that:

One should be able to perform a partial truncation of Eisenstein series by chopping away parts of the constant terms that one knows to behave well, leaving behind parts that are not known to behave well but which are squareintegrable.

For the groups of rank two that we have looked at earlier in this paper, this amounts to the observation that I have already made, that the bars 0 in my diagrams, as well as certain reflections of them, lie in the domain of squareintegrability. Whether this idea remains viable for all Eisenstein series associated to square-integrable residues seems to involve a complicated problem in geometric combinatorics, one I have verified by computer in many cases.+

\section{REFERENCES}

[1] Bill Casselman, 'Introduction to the Schwartz space of $\Gamma \backslash G$ ', Canadian Journal of Mathematics XL (1989), 285-320.

[2] _ 'Harmonic analysis of the Schwartz space of $\Gamma \backslash S L_{2}(\mathbb{R})$ ', pages 163-192 in Contributions to automorphic forms, geometry, and number theory, the proceedings of a conference to honor Joe Shalika, Johns Hopkins, 2004.

[3] Yves Colin de Verdière, 'Une nouvelle démonstration du prolongement méromorphe des séries d'Eisenstein', Comptes Rendues de l'Académie des Sciences de Paris 293 (1981), 361-363.

[4] Jens Franke, 'Harmonic analysis in weighted $L^{2}$-spaces', Annales de l'École Normale Supérieure 31 (1998), 181-279.

[5] ' 'On the singularities of residual Eisenstein series', Inventiones Mathematicae 138 (1999), 307-317.

[6] James E. Humphries, Reflection groups and Coxeter groups, Cambridge Studies in advanced mathematics 29, Cambridge University Press, 1990.

[7] Bertram Kostant, 'The principal three-dimensional subgroup and the Betti numbers of a complex simple Lie group', American Journal of Mathematics 81 (1959), 973-1032.

[8] Robert P. Langlands, 'Eisenstein series', in Algebraic groups and discontinuous subgroups, Proceedings of symposia in pure mathematics IX , American Mathematical Society, 1966.

[9] , On the functional equations satisfied by Eisenstein series, Lecture Notes in Mathematics 544, Springer, 1976.

[10] Werner Müller, 'The trace class conjecture in the theory of automorphic forms', Annals of Mathematics 130 (1989), 473-529.

Bill Casselman

Department of Mathematics

University of British Columbia

Vancouver, Canada

E-mail: cass@math.ubc.ca 